\title{
Modularity in the Semilattice of $\omega$-Words
}

\author{
Jānis Buls and Edmunds Cers \\ Department of Mathematics, University of Latvia, 19 Rainis Boulevard, Riga 1586, Latvia \\ Correspondence should be addressed to Jānis Buls; buls@fmf.lu.lv
}

Received 31 August 2013; Accepted 6 December 2013; Published 18 May 2014

Academic Editor: Teturo Kamae

Copyright (C) 2014 J. Buls and E. Cers. This is an open access article distributed under the Creative Commons Attribution License, which permits unrestricted use, distribution, and reproduction in any medium, provided the original work is properly cited.

\begin{abstract}
A partial ordering of $\omega$-words can be introduced with regard to whether an $\omega$-word can be transformed into another by a Mealy machine. It is known that the poset of $\omega$-words that is introduced by this ordering is a join-semilattice. The width of this joinsemilattice has the power of continuum while the depth is at least $\aleph_{0}$. We have created a technique for proving that powercharacteristic $\omega$-words are incomparable. We use this technique to show that this join-semilattice is not modular.
\end{abstract}

\section{Introduction}

Infinite words ( $\omega$-words) provide a fertile ground of research and many classes of $\omega$-words are known. While closure properties of some classes of $\omega$-words have been studied extensively (see, e.g., [1-3]), we are interested in the general algebraic structure of $\omega$-words. Mealy machines are a simple model of a word transforming automaton with the beneficial property of always transforming an $\omega$-word into an $\omega$-word. A partial ordering is defined on $\omega$-words by the existence of a Mealy machine transforming one word into another (we write $x \neg y$ if such a machine exists). When both $x \rightarrow y$ and $y \neg x$ are true, we say that $x$ and $y$ are machine equivalent. A class $\mathbb{R}$ of $\omega$-words is called machine invariant if $\mathbb{\Re}$ contains all possible transformations of its elements.

Buls [4] has shown that machine invariant classes of $\omega$-words form a completely distributive lattice. Belovs [5] showed that the poset of machine equivalent classes of $\omega$ words is a join-semilattice and that the width of this joinsemilattice has the power of continuum while the depth is at least $\aleph_{0}$. We show in this paper that this join-semilattice is not modular.

\section{Preliminaries}

Let $A$ be a finite, nonempty set and $A^{*}$ the free monoid generated by $A$. Call $A$ an alphabet, its elements letters, and the elements of $A^{*}$ finite words. The identity element of $A^{*}$ is called the empty word and is denoted by $\lambda$.
Let $w$ be a word over a finite and non-empty alphabet $A$. We denote the length of $w$ by $|w|$. Similarly, the cardinality of $A$ is denoted by $|A|$. The concatenation of the words $u, v$ is denoted by $u \# v$ or simply $u v$. Define $w^{0}=\lambda$ and for all $i w^{i+1}=w^{i} w$. We say that $u$ (resp., $v$ ) is a prefix (resp., suffix) of $u v$. Denote by $\operatorname{Pref}(w)$ and $\operatorname{Suff}(w)$ the respective sets of prefixes and suffixes of $w$. Let $\overline{k, n}$ denote the set of integers $\{k, k+1, k+2, \ldots, n\}:$

$$
\mathbb{N}=\{0,1,2, \ldots, n, \ldots\}
$$

Call a total map $x: \mathbb{N} \rightarrow A$ an (indexed) $\omega$-word of the alphabet $A$. For any $i \geq 0$ define $x_{i}=x(i)$ and write

$$
\begin{gathered}
x=\left(x_{i}\right)=x_{0} x_{1} \cdots x_{n} \cdots \\
x(k+1, n+1)=x[k, n+1)=x[k, n]=x_{k} x_{k+1} \ldots x_{n-1} x_{n} .
\end{gathered}
$$

The set of all $\omega$-words over $A$ is denoted by $A^{\omega}$. We say that $y$ is a prefix of $x$, if there exists an integer $k$ such that $y=x[0, k]$. An $\omega$-word $x$ is called ultimately periodic if there exist integers $p \geq 0$ and $T>0$ such that $x_{i}=x_{i+T}$ for all $i \geq p$. In this case $p$ is called preperiod and T a period of $x$. An ultimately periodic $\omega$-word with a preperiod of zero is called periodic. We say a finite word $w$ is (ultimately) periodic if it is a prefix of some (ultimately) periodic $\omega$-word. We recall the important periodicity theorem due to Fine and Wilf [6]. 
Theorem 1. Let $w$ be a word having periods $p$ and $q$ and denote by $\operatorname{gcd}(p, q)$ the greatest common divisor of $p$ and $q$. If $|w| \geq$ $p+q-\operatorname{gcd}(p, q)$, then $w$ has also the period $\operatorname{gcd}(p, q)$.

Corollary 2. Let uv and $v w$ be words having periods, respectively, $p$ and $q$. If $|v| \geq p+q-\operatorname{gcd}(p, q)$, then uvw has the period $\operatorname{gcd}(p, q)$.

This is almost folklore in combinatorics on words. Nevertheless, for the sake of completeness, we will give the proof of this corollary.

Proof. Since $|v| \geq p+q-\operatorname{gcd}(p, q)$, then (Theorem 1) $v$ has the period $\operatorname{gcd}(p, q)$.

(i) At first we will prove by induction on $|u|$ that $u v$ has the period $\operatorname{gcd}(p, q)$. Let $v=v_{1} v_{2} \cdots v_{k}$. If $|u|=1$, then $u=v_{0}$ for some letter. Since the period of $u v$ is $p$ then $v_{0}=v_{p}$.

Notice that $\operatorname{gcd}(p, q)$ divides $p$. Since the period of $v$ is $\operatorname{gcd}(p, q)$, then $v_{p}=v_{\operatorname{gcd}(p, q)}$. Hence, $v_{0}=v_{\operatorname{gcd}(p, q)}$.

(ii) If $|u|>1$, then $u$ can be represented as concatenation $a u^{\prime}=u$, where $\left|u^{\prime}\right|+1=|u|$. By assumption the period of $u^{\prime} v$ is $\operatorname{gcd}(p, q)$. Now from (i) follows that the period of $a u^{\prime} v$ is $\operatorname{gcd}(p, q)$ too. We have completed the inductive step.

Now we shall prove by induction on $|w|$ that $v w$ has the period $\operatorname{gcd}(p, q)$.

(iii) If $|w|=1$, then $w=v_{k+1}$ for some letter. Since the period of $v w$ is $q$, then $v_{k+1-q}=v_{k+1}$. Notice

$$
k+1-\operatorname{gcd}(p, q) \equiv k+1-q, \quad(\bmod \operatorname{gcd}(p, q)) .
$$

Hence, $v_{k+1-\operatorname{gcd}(p, q)}=v_{k+1-q}=v_{k+1}$.

(iv) If $|w|>1$, then $w$ can be represented as concatenation $w^{\prime} b=w$, where $\left|w^{\prime}\right|+1=|w|$. By assumption the period of $v w^{\prime}$ is $\operatorname{gcd}(p, q)$. Now from (iii) follows that the period of $v w^{\prime} b$ is $\operatorname{gcd}(p, q)$ too. We have completed the inductive step.

A 3-sorted algebra $V=\left\langle Q, A, B ; q_{0}, \circ, *\right\rangle$ is called an initial Mealy machine if $Q, A, B$ are finite, nonempty sets, called the set of states, the input alphabet, and the output alphabet, respectively, $q_{0} \in Q$ is called the initial state, $\circ$ : $Q \times A \rightarrow Q$ is a total function called the transition function, and $*: Q \times A \rightarrow B$ is a total surjective function called the output function. We write $\langle Q, A, B ; \circ, *\rangle$ or even $\left\langle Q, A, B ; q_{0}\right\rangle$ if there is no danger of confusion. The mappings $\circ$ and $*$ are extended to $Q \times A^{*}$ by defining

$$
\begin{gathered}
q \circ \lambda=q, \quad q \circ(u a)=(q \circ u) \circ a, \\
q * \lambda=\lambda, \quad q *(u a)=(q * u) \#((q \circ u) * a),
\end{gathered}
$$

for all $q \in Q,(u, a) \in A^{*} \times A$. Henceforth, we shall omit parentheses if there is no danger of confusion. So, for example, we will write $q \circ u * a$ instead of $(q \circ u) * a$. Let $(x, y) \in A^{\omega} \times B^{\omega}$. If for some Mealy machine $V$ : for all $n y[0, n]=q_{0} * x[0, n]$, we say that $V$ transforms $x$ to $y$ and write $y=q_{0} * x$ or $x \stackrel{V}{\rightarrow} y$. We write $x \neg y$ if there exists $V$ such that $x \stackrel{V}{\neg} y$; otherwise, we write $x \neq y$. We write $x \rightleftharpoons y$ if $x \rightarrow y$ and $y \rightarrow x$ and say that $x$ and $y$ are machine equivalent.

Given the integers $a_{1}, a_{2}, \ldots, a_{n}$, let $\operatorname{lcm}\left(a_{1}, a_{2}, \ldots, a_{n}\right)$ denote the least common multiple of $a_{1}, a_{2}, \ldots, a_{n}$. Given a
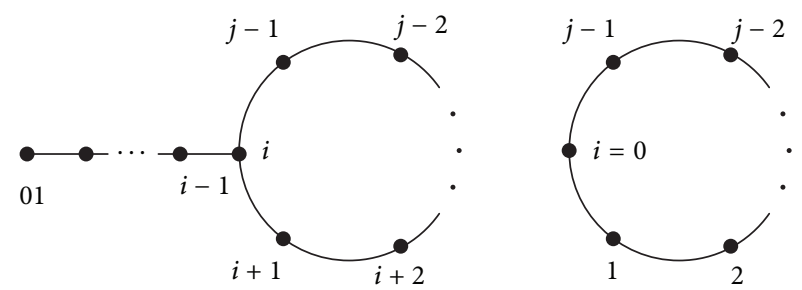

Figure 1: Indices and periods.

number $x \in \mathbb{R}$, denote by $\lfloor x\rfloor$ the greatest integer less than or equal to $x$ and by $\lceil x\rceil$ the least integer greater than or equal to $x$.

\section{Machine Transformations of Power-Characteristic $\omega$-Words}

Definition 3. We will call the $\omega$-word ${ }^{\zeta} x \in\{0,1\}^{\omega}$ the characteristic word of the power $\zeta$ if

$$
\zeta x(n)= \begin{cases}1, & \text { if } \exists k \in \mathbb{N}, n=k^{\zeta} \\ 0, & \text { otherwise. }\end{cases}
$$

For example, ${ }^{2} x=110010000100 \ldots$ is the characteristic word of the squares.

Convention. Henceforth, we assume that $\zeta \geq 2$ and it is a natural number.

More generally, let $f: \mathbb{N} \rightarrow \mathbb{N}$ be any total increasing function; then

$$
{ }^{f} x(n)= \begin{cases}1, & \text { if } \exists k \in \mathbb{N}, n=f(k), \\ 0, & \text { otherwise. }\end{cases}
$$

Let $V=\langle Q,\{0,1\},\{0,1\} ; \circ, *\rangle$ be a Mealy machine, where

$$
Q=\left\{q_{1}^{\prime}, q_{2}^{\prime}, \ldots, q_{b}^{\prime}\right\} .
$$

Applying the pigeonhole principle, we can state that for every $q \in Q$ there is a least integer $i \geq 0$ such that $q \circ 0^{i}=q \circ 0^{j}$ for some $i<j$. The integer $i$ is called the index of $q$, and $j-i$ is called the period of $q$. We can visualize this as the diagrams (see Figure 1).

Claim 1. If $a_{s}$ is the index and $c_{s}$ is the period of $q_{s}^{\prime}$,

$$
a_{s} \leq m_{1}<m_{2}, \quad m_{1} \equiv m_{2} \quad\left(\bmod c_{s}\right),
$$

then

$$
q_{s}^{\prime} \circ 0^{m_{1}}=q_{s}^{\prime} \circ 0^{m_{2}} .
$$

Claim 2. If $\max \left(a_{1}, a_{2}, \ldots, a_{b}\right) \leq m_{1}<m_{2}$ and $m_{1} \equiv m_{2}$ $(\bmod m)$, where

$$
m=\operatorname{lcm}\left(c_{1}, c_{2}, \ldots, c_{b}\right),
$$


then

$$
\forall q \in Q, \quad q \circ 10^{m_{1}}=q \circ 10^{m_{2}} .
$$

Let $\alpha(X)$ be an integer polynomial; that is, $\alpha(X) \in \mathbb{Z}[X]$. The following theorem is known from elementary number theory.

Theorem 4. If $i \equiv j(\bmod m)$, then $\alpha(i) \equiv \alpha(j)(\bmod m)$.

If we take $\alpha_{k}=(k+1)^{\zeta}-k^{\zeta}-1$, then we can express

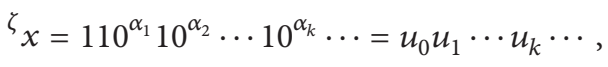

where $u_{k}=10^{\alpha_{k}}$. Hence, ${ }^{\zeta} x\left[0, k^{\zeta}\right)=u_{0} u_{1} \cdots u_{k-1}$.

Corollary 5. If $i \equiv j(\bmod m)$, then $\alpha_{i} \equiv \alpha_{j}(\bmod m)$.

Let

$$
\begin{gathered}
w_{0}=u_{0}, \\
w_{k+1}=w_{k} u_{k+1} .
\end{gathered}
$$

Then

$$
\zeta_{x}\left[0, k^{\zeta}\right)=u_{0} u_{1} \cdots u_{k-1}=w_{k-1} .
$$

Let $q \in Q$. We define a sequence

$$
q_{0}, q_{1}, \ldots, q_{k}, \ldots
$$

where $q_{k}=q \circ w_{k}$.

Corollary 6. The sequence $q_{0}, q_{1}, \ldots, q_{k}, \ldots$ is ultimately periodic.

Proof. Let $m=\operatorname{lcm}\left(c_{1}, c_{2}, \ldots, c_{b}\right)$. There exists $n$ such that

$$
\left|u_{m n}\right|>\max \left(a_{1}, a_{2}, \ldots, a_{b}\right) \text {. }
$$

Now consider the sequence $q_{m n}, q_{m(n+1)}, \ldots, q_{m(n+b)}$. Since $|\overline{0, b}|=b+1>|Q|$, then-by the pigeonhole principle-there must exist two equal states

$$
q_{m(n+i)}=q_{m(n+j)}, \quad \text { with } 0 \leq i<j \leq b .
$$

Hence

$$
\begin{aligned}
& q_{m(n+i)+1}=q_{m(n+i)} \circ u_{m(n+i)+1}=q_{m(n+j)} \circ u_{m(n+i)+1} \\
& \underset{\text { Claim 2 }}{=} q_{m(n+j)} \circ u_{m(n+j)+1}=q_{m(n+j)+1} .
\end{aligned}
$$

The rest follows by induction.

The following lemma is very easy, but it turns out to be useful.

Lemma 7. Let $V=\left\langle Q, A, B ; q_{0}\right\rangle$ be a Mealy machine. If $|Q|=$ $m$ and $q_{0} * 0^{s}=w$, then there exists $\varkappa$ such that

$$
w=u v^{\varkappa} \dot{v}, \quad \text { where }|u|+|v| \leq m \text { and } \dot{v} \in \operatorname{Pref}(v) .
$$

Proof. (i) If $s \leq m$, then $|w|=\left|0^{s}\right|=s \leq m$, and we can choose $u=w, v=\dot{v}=\lambda$.

(ii) Let $s>m$ and $q_{0}, q_{1}, \ldots, q_{m}$ states, where

$$
\forall i \in \overline{0, m} \quad q_{i}=q_{0} \circ 0^{i} .
$$

Since $|\overline{0, m}|=m+1>|Q|$, then-by the pigeonhole principle - there must exist two equal states; namely, there exist $i$ and $j, 0 \leq i<j \leq m$, such that

$$
q_{i}=q_{j}
$$

Putting

$$
\begin{gathered}
u=q_{0} * 0^{i}, \quad v=q_{i} * 0^{j-i}, \\
\varkappa=\left\lfloor\frac{s-i}{j-i}\right\rfloor, \quad \dot{v}=q_{i} * 0^{s-i-\varkappa(j-i)},
\end{gathered}
$$

then $|u|+|v|=\left|0^{i}\right|+\left|0^{j-i}\right|=i+(j-i)=j \leq m$ and $w=$ $u v^{x} \dot{v}$.

Proposition 8. If ${ }^{f} x \rightarrow y,{ }^{\zeta} x \rightarrow y$ and

$$
\forall \varkappa \exists a k \quad f(k) \leq a^{\zeta}<(a+\varkappa)^{\zeta} \leq f(k+1),
$$

then $y$ is ultimately periodic.

Proof. Since ${ }^{\zeta} x \rightarrow y$ and ${ }^{f} x \rightarrow y$, there exist Mealy machines

$$
V=\langle Q,\{0,1\}, B ; q, \circ, *\rangle, \quad V^{\prime}=\left\langle Q^{\prime},\{0,1\}, B ; q^{\prime}, \circ^{\prime}, *^{\prime}\right\rangle
$$

such that ${ }^{\zeta} x \stackrel{V}{\rightarrow} y$ and ${ }^{f} x \stackrel{V^{\prime}}{\rightarrow} y$.

(i) First, we express ${ }^{\zeta} x=u_{0} u_{1} \cdots u_{n} \cdots$ with

$$
u_{n}=10^{\alpha_{n}}, \quad \alpha_{n}=(n+1)^{\zeta}-n^{\zeta}-1,
$$

and look at the sequence $q_{0}, q_{1}, \ldots, q_{n}, \ldots$, where

$$
q_{n}=q \circ\left(u_{0} u_{1} \cdots u_{n}\right) .
$$

We have shown (Corollary 6) that the sequence $q_{0}, q_{1}, \ldots, q_{n}, \ldots$ is ultimately periodic. Assume its period is $T$ and the preperiod $p$.

(ii) By assumption (see (23)) we can choose integers $k$ and $a$ such that

$$
f(k) \leq a^{\zeta}<(a+T+1)^{\zeta} \leq f(k+1)
$$

and, moreover, $a>p+7$ and $(a+1)^{\zeta}-a^{\zeta}>3 \cdot \max \left(|Q|,\left|Q^{\prime}\right|\right)+$ 7. Now, ${ }^{f} x(f(k), f(k+1))$ is a word of the form $0^{d}$, and thus (by Lemma 7)

$$
y(f(k), f(k+1))
$$

must be ultimately periodic with both its period and preperiod not greater than $\left|Q^{\prime}\right|$. We denote this preperiod by $p^{\prime}$ and the least period by $T^{\prime}$. Since

$$
p^{\prime} \leq\left|Q^{\prime}\right|<(a+1)^{\zeta}-a^{\zeta}-7,
$$


then $y\left[(a+1)^{\zeta},(a+1+T)^{\zeta}\right)$ is periodic with the period $T^{\prime}$. Notice that

$$
\begin{aligned}
y & {\left[(a+i)^{\zeta},(a+i+1)^{\zeta}\right) } \\
& =q_{a+i-1} * \zeta \times\left[(a+i)^{\zeta},(a+i+1)^{\zeta}\right)=q_{a+i-1} * 10^{\alpha_{a+i}}
\end{aligned}
$$

and that the sequence of states $q_{a}, q_{a+1}, q_{a+2}, \ldots, q_{a+T}, \ldots$ is also periodic. Therefore

$$
\begin{aligned}
y & {\left[(a+i+T)^{\zeta},(a+i+1+T)^{\zeta}\right) } \\
& =q_{a+i-1+T} * \zeta^{\zeta}\left[(a+i+T)^{\zeta},(a+i+1+T)^{\zeta}\right) \\
& =q_{a+i-1+T} * 10^{\alpha_{a+i+T}}=q_{a+i-1} * 10^{\alpha_{a+i+T}}=\dot{u} \dot{v} \dot{w},
\end{aligned}
$$

where $|\dot{u}|=|\dot{v}|=\max \left(|Q|,\left|Q^{\prime}\right|\right)$. So we have two periodic words $\dot{u} \dot{v}$ and $\dot{v} \dot{w}$. Hence by Corollary 2

$$
y\left[(a+i+T)^{\zeta},(a+i+1+T)^{\zeta}\right)
$$

is periodic with period $T^{\prime}$. Therefore we can conclude that $y\left[(a+i)^{\zeta},(a+i+1)^{\zeta}\right)$ is periodic with period $T^{\prime}$ for all $i>0$; besides, $T^{\prime}$ is the least period for all $i>0$.

(iii) Let $X>a$ and $\mu=\operatorname{lcm}\left(T, T^{\prime}\right)$. Then

$$
\begin{aligned}
(X+\mu)^{\zeta}-X^{\zeta} & =\sum_{j=0}^{\zeta}\left(\begin{array}{l}
\zeta \\
j
\end{array}\right) \mu^{j} X^{\zeta-j}-X^{\zeta} \\
& =\mu \sum_{j=1}^{\zeta}\left(\begin{array}{l}
\zeta \\
j
\end{array}\right) \mu^{j-1} X^{\zeta-j}=\mu P(X),
\end{aligned}
$$

where

$$
P(X)=\sum_{j=1}^{\zeta}\left(\begin{array}{l}
\zeta \\
j
\end{array}\right) \mu^{j-1} X^{\zeta-j} .
$$

We have shown in (ii) that $y\left[X^{\zeta},(X+1)^{\zeta}\right)$ is periodic. Therefore there is a $v$ such that

$$
y\left[X^{\zeta},(X+1)^{\zeta}\right)=v^{r} v^{\prime}
$$

where $|v|=T^{\prime}$ and $v^{\prime} \in \operatorname{Pref}(v)$. Since $T$ divides $\mu$, then $q_{X-1}=q_{X-1+\mu}$. But then

$$
\begin{aligned}
& y\left[(X+\mu)^{\zeta},(X+\mu+1)^{\zeta}\right) \\
& =q_{X+\mu-1} *{ }^{\zeta} x\left[(X+\mu)^{\zeta},(X+\mu+1)^{\zeta}\right) \\
& =q_{X-1} *{ }^{\zeta} x\left[(X+\mu)^{\zeta},(X+\mu+1)^{\zeta}\right) \\
& =q_{X-1} * 10^{\alpha_{X+\mu}}=v^{r^{\prime}} v^{\prime \prime},
\end{aligned}
$$

for some number $r^{\prime}$ and $v^{\prime \prime} \in \operatorname{Pref}(v)$. It follows from (33) that

$$
(X+\mu+1)^{\zeta}-(X+\mu)^{\zeta} \equiv(X+1)^{\zeta}-X^{\zeta} \quad\left(\bmod T^{\prime}\right)
$$

and therefore $v^{\prime}=v^{\prime \prime}$. (iv) Finally, we can select integers $\breve{k}, \breve{a}$ such that $k<\breve{k}$ and

$$
f(\breve{k}) \leq \breve{a}^{\zeta}<(\breve{a}+\mu+1)^{\zeta} \leq f(\breve{k}+1) .
$$

Now we repeat the proof from (ii). So we can conclude that there is the least period $T^{\prime \prime} \leq\left|Q^{\prime}\right|$ of the word $y\left[(\breve{a}+1)^{\zeta}\right.$, $\left.(\breve{a}+1+\mu)^{\zeta}\right)$. A period of

$$
y\left[(\breve{a}+1)^{\zeta},(\breve{a}+2)^{\zeta}\right)
$$

is $T^{\prime}$ too. Hence (Theorem 1) $T^{\prime \prime}=T^{\prime}$. Denote $y\left[(\breve{a}+1)^{\zeta}\right.$, $\left.(\breve{a}+1)^{\zeta}+T^{\prime}\right)=u$. Since (from formula $\left.(33)\right)$

$$
(\breve{a}+1+\mu)^{\zeta}-(\breve{a}+1)^{\zeta} \equiv 0 \quad\left(\bmod T^{\prime}\right),
$$

there is an integer $s$ such that $y\left[(\breve{a}+1)^{\zeta},(\breve{a}+1+\mu)^{\zeta}\right)=u^{s}$. As it was shown in (iii) we can choose $s_{1}^{\prime}, s_{2}^{\prime}$ such that

$$
\begin{gathered}
y\left[(\breve{a}+1)^{\zeta},(\breve{a}+2)^{\zeta}\right)=u^{s_{1}^{\prime}} u^{\prime}, \\
y\left[(\breve{a}+1+\mu)^{\zeta},(\breve{a}+2+\mu)^{\zeta}\right)=u^{s_{2}^{\prime}} u^{\prime} .
\end{gathered}
$$

But then $y\left[(\breve{a}+1)^{\zeta},(\breve{a}+2+\mu)^{\zeta}\right)=u^{s} u^{s{ }^{\prime}} u^{\prime}$, which means that

$$
y\left[(\breve{a}+1)^{\zeta},(\breve{a}+2+\mu)^{\zeta}\right)
$$

is periodic with the period $T^{\prime}$. Now suppose that $y\left[(\breve{a}+1)^{\zeta}\right.$, $\left.(\breve{a}+n)^{\zeta}\right)=u^{\sigma} \breve{u}$, where $n>\mu+1$ and $\breve{u} \in \operatorname{Pref}(u)$. Then there exists such $\breve{v} \in \operatorname{Suff}(u)$ that $\breve{u} \breve{v}=u$. From (see formula (33)) $(\breve{a}+n)^{\zeta}-(\breve{a}+n-\mu)^{\zeta} \equiv 0(\bmod T)^{\prime}$, we can conclude that

$$
\breve{v} \in \operatorname{Pref}\left(y\left[(\breve{a}+n-\mu)^{\zeta},(\breve{a}+n-\mu+1)^{\zeta}\right)\right) .
$$

It follows from what we have shown in (iii) that there are $\sigma_{1}, \sigma_{2}$ such that

$$
\begin{gathered}
y\left[(\breve{a}+n-\mu)^{\zeta},(\breve{a}+n-\mu+1)^{\zeta}\right)=v^{\sigma_{1}} v^{\prime}, \\
y\left[(\breve{a}+n)^{\zeta},(\breve{a}+n+1)^{\zeta}\right)=v^{\sigma_{2}} v^{\prime}
\end{gathered}
$$

with $|v|=T^{\prime}$ and $v^{\prime} \in \operatorname{Pref}(v)$. But then $v=\breve{v} \breve{u}$ and

$$
\begin{aligned}
y\left[(\breve{a}+1)^{\zeta},(\breve{a}+n+1)^{\zeta}\right) & =u^{\sigma} \breve{u} v^{\sigma_{2}} v^{\prime}=u^{\sigma} \breve{u}(\breve{v} \breve{u})^{\sigma_{2}} v^{\prime} \\
& =u^{\sigma}(\breve{u} \breve{v})^{\sigma_{2}} \breve{u} v^{\prime}=u^{\sigma+\sigma_{2}} \breve{u} v^{\prime} .
\end{aligned}
$$

This means that $y\left[(\breve{a}+1)^{\zeta},(\breve{a}+n+1)^{\zeta}\right)$ is periodic with period $T^{\prime}$. Now, by induction, we have $y\left[(\breve{a}+1)^{\zeta},(\breve{a}+i)^{\zeta}\right]$ is periodic with the period $T^{\prime}$ for any $i>1$. Hence, $y$ is ultimately periodic.

\section{Modularity in the Semilattice of $\omega$-Words}

Our main object of investigation is the machine poset of infinite words. In order to avoid some set-theoretical problems, we make some assumptions. Let us take the set 
$\mathfrak{N}=\bigcup_{k=0}^{\infty}(\overline{0, k})^{\omega}$. We shall assume that the states of the involved Mealy machines as well as their input and output alphabets all are from the set $\mathbb{N}$. If another input or output alphabet $A$ is used, we assume that there exists a bijection $\beta: A \rightarrow \overline{0,|A|-1}$ and that this bijection is applied to the input or output word, respectively.

We suppose that the reader is familiar with the basic notions of ordered sets [7]. If $\rightarrow$ is used as an algebraic relation on $\mathfrak{N}$, then the algebraic structure $\langle\mathfrak{N}, \rightarrow\rangle$ defines a preorder [5], while the quotient set $\widetilde{\mathfrak{N}}=\mathfrak{N} / \rightleftharpoons$ becomes the ordered set $\langle\mathfrak{N}, \rightarrow\rangle$. It has been shown that this poset $\widetilde{\mathfrak{N}}$ is a join-semilattice [5], where the join $\left[\left(x_{i}\right)\right] \vee\left[\left(y_{i}\right)\right]=\left[\left(x_{i}, y_{i}\right)\right]$.

Definition 9. A join-semilattice $\langle D, \leq\rangle$ is distributive when

$\forall x a b\left(x \leq a \vee b \Longrightarrow \exists a^{\prime} b^{\prime}\left(a^{\prime} \leq a \& b^{\prime} \leq b \& x=a^{\prime} \vee b^{\prime}\right)\right)$

A join-semilattice $\langle D, \leq\rangle$ is modular when

$$
\forall x a b\left(a \leq x \leq a \vee b \Longrightarrow \exists b^{\prime} \leq b\left(x=a \vee b^{\prime}\right)\right)
$$

Theorem 10. The join-semilattice $\langle\widetilde{\mathfrak{N}}, \rightarrow\rangle$ is not modular.

Proof. We start by showing that ${ }^{2} x \vee^{4} x \rightarrow x^{\prime}$, where

$$
x^{\prime}(n)= \begin{cases}1, & \text { if } \exists k \in \mathbb{N}, n=k^{4}, \\ 1, & \text { if } \exists k \in \mathbb{N}, n=\left(k^{2}+1\right)^{2} \\ 0, & \text { otherwise. }\end{cases}
$$

By definition, $\left({ }^{2} x \vee^{4} x\right)(n)=\left({ }^{2} x(n),{ }^{4} x(n)\right)$. Define the Mealy machine

$$
V=\left\langle\left\{q_{0}, q_{1}, q_{2}\right\},\left\{\left(\begin{array}{l}
0 \\
0
\end{array}\right),\left(\begin{array}{l}
0 \\
1
\end{array}\right),\left(\begin{array}{l}
1 \\
0
\end{array}\right),\left(\begin{array}{l}
1 \\
1
\end{array}\right)\right\},\{0,1\} ; q_{0}, \circ, *\right\rangle
$$

by

$$
\begin{aligned}
q_{1}=q_{0} \circ\left(\begin{array}{l}
0 \\
0
\end{array}\right)=q_{0} \circ\left(\begin{array}{l}
0 \\
1
\end{array}\right)=q_{0} \circ\left(\begin{array}{l}
1 \\
0
\end{array}\right)=q_{0} \circ\left(\begin{array}{l}
1 \\
1
\end{array}\right) \\
=q_{1} \circ\left(\begin{array}{l}
0 \\
0
\end{array}\right)=q_{1} \circ\left(\begin{array}{l}
0 \\
1
\end{array}\right)=q_{1} \circ\left(\begin{array}{l}
1 \\
0
\end{array}\right)=q_{2} \circ\left(\begin{array}{l}
1 \\
0
\end{array}\right), \\
q_{2}=q_{1} \circ\left(\begin{array}{l}
1 \\
1
\end{array}\right)=q_{2} \circ\left(\begin{array}{l}
0 \\
0
\end{array}\right)=q_{2} \circ\left(\begin{array}{l}
0 \\
1
\end{array}\right)=q_{2} \circ\left(\begin{array}{l}
1 \\
1
\end{array}\right), \\
0=q_{1} *\left(\begin{array}{l}
0 \\
0
\end{array}\right)=q_{1} *\left(\begin{array}{l}
0 \\
1
\end{array}\right)=q_{1} *\left(\begin{array}{l}
1 \\
0
\end{array}\right) \\
=q_{2} *\left(\begin{array}{l}
0 \\
0
\end{array}\right)=q_{2} *\left(\begin{array}{l}
0 \\
1
\end{array}\right)=q_{2} *\left(\begin{array}{l}
1 \\
1
\end{array}\right), \\
1=q_{0} *\left(\begin{array}{l}
0 \\
0
\end{array}\right)=q_{0} *\left(\begin{array}{l}
0 \\
1
\end{array}\right)=q_{0} *\left(\begin{array}{l}
1 \\
0
\end{array}\right) \\
=q_{0} *\left(\begin{array}{l}
1 \\
1
\end{array}\right)=q_{1} *\left(\begin{array}{l}
1 \\
1
\end{array}\right)=q_{2} *\left(\begin{array}{l}
1 \\
0
\end{array}\right) .
\end{aligned}
$$

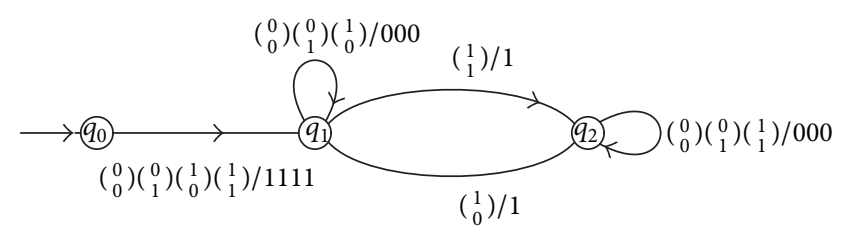

FIgURE $2:{ }^{2} x \vee^{4} x \stackrel{V}{\rightarrow} x^{\prime}$

We illustrate this by the diagram in Figure 2. It follows straightforwardly from the construction that ${ }^{2} x \vee^{4} x \stackrel{V}{\rightarrow}$ $x^{\prime} \rightarrow{ }^{4} x$. Now suppose that there exists $y$ such that ${ }^{2} x \rightarrow y$ and $x^{\prime} \rightleftharpoons{ }^{4} x \vee y$. But then $x^{\prime} \rightarrow y$ too. Notice that $x^{\prime}={ }^{g} x$ for

$$
g(k)= \begin{cases}0, & \text { if } k=0 \\ \frac{(k+1)^{4}}{16}, & \text { if } k \text { is odd } \\ \left(\frac{k^{2}}{4}+1\right)^{2}, & \text { if } k \text { is even }\end{cases}
$$

Hence, by Proposition $8 y$ is ultimately periodic. But if so, then ${ }^{4} x \vee y \rightleftharpoons{ }^{4} x$. However this is a contradiction because then $x^{\prime} \rightleftharpoons{ }^{4} x$

Corollary 11 (see [8]). The join-semilattice $\langle\widetilde{\mathfrak{N}}, \rightarrow\rangle$ is not distributive.

We recall that every distributive join-semilattice is modular.

\section{Conclusion}

We agree that it is not sufficient to state that $\widetilde{\mathfrak{N}}$ is very complicated as it was done in Wikipedia writing review about Turing degrees [9]. Nevertheless it is clear that $\widetilde{\mathfrak{N}}$ is not modular and that there is no classical algebraic notion that describes such semilattice axiomatically at this moment. So we have challenge to extract axioms for semilattice $\widetilde{\mathfrak{N}}$.

\section{Conflict of Interests}

The authors declare that there is no conflict of interests regarding the publication of this paper.

\section{Acknowledgment}

The useful suggestions of two referees are gratefully acknowledged.

\section{References}

[1] A. Cobham, "Uniform tag sequences," Mathematical Systems Theory, vol. 6, pp. 164-192, 1972.

[2] F. M. Dekking, "Iteration of maps by an automaton," Discrete Mathematics, vol. 126, no. 1-3, pp. 81-86, 1994. 
[3] A. Muchnik, A. Semenov, and M. Ushakov, "Almost periodic sequences," Theoretical Computer Science, vol. 304, no. 1-3, pp. 1-33, 2003.

[4] J. Buls, Machine Invariant Classes, vol. 27, TUCS General Publications, 2003.

[5] A. Belovs, "Some algebraic properties of machine poset of infinite words," Theoretical Informatics and Applications, vol. 42, no. 3, pp. 451-466, 2008.

[6] N. J. Fine and H. S. Wilf, "Uniqueness theorems for periodic functions," Proceedings of the American Mathematical Society, vol. 16, pp. 109-114, 1965.

[7] G. Birkhoff, Lattice Theory, Providence, RI, USA, 1967.

[8] J. Buls and E. Cers, "Distributivity in the semilattice of $\omega$ words," in Contributions to General Algebra 19, pp. 13-21, Heyn, Klagenfurt, Austria, 2010.

[9] Turing degree, Wikipedia, November 2013, http://en.wikipedia .org/wiki/Turing_degree. 


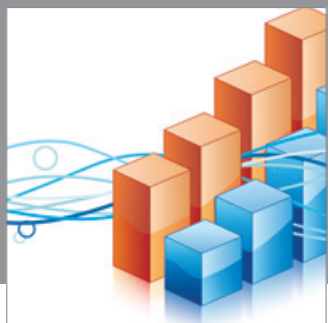

Advances in

Operations Research

mansans

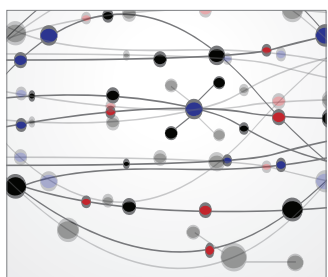

The Scientific World Journal
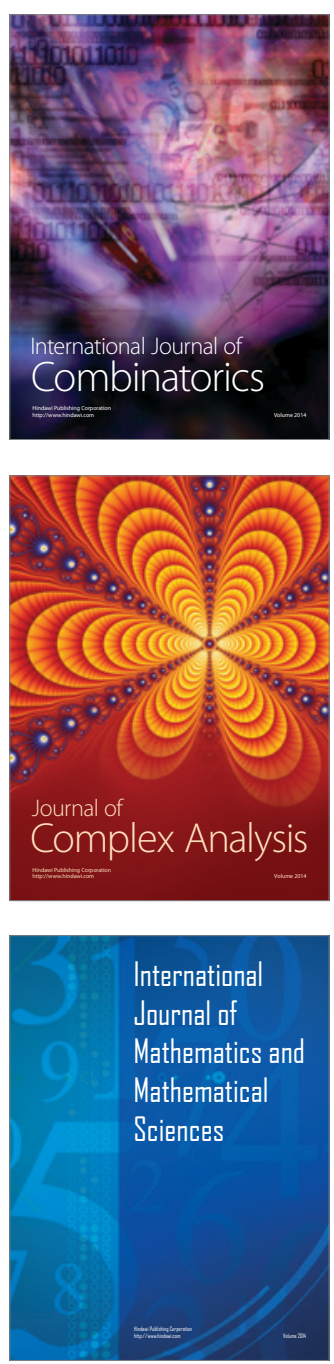
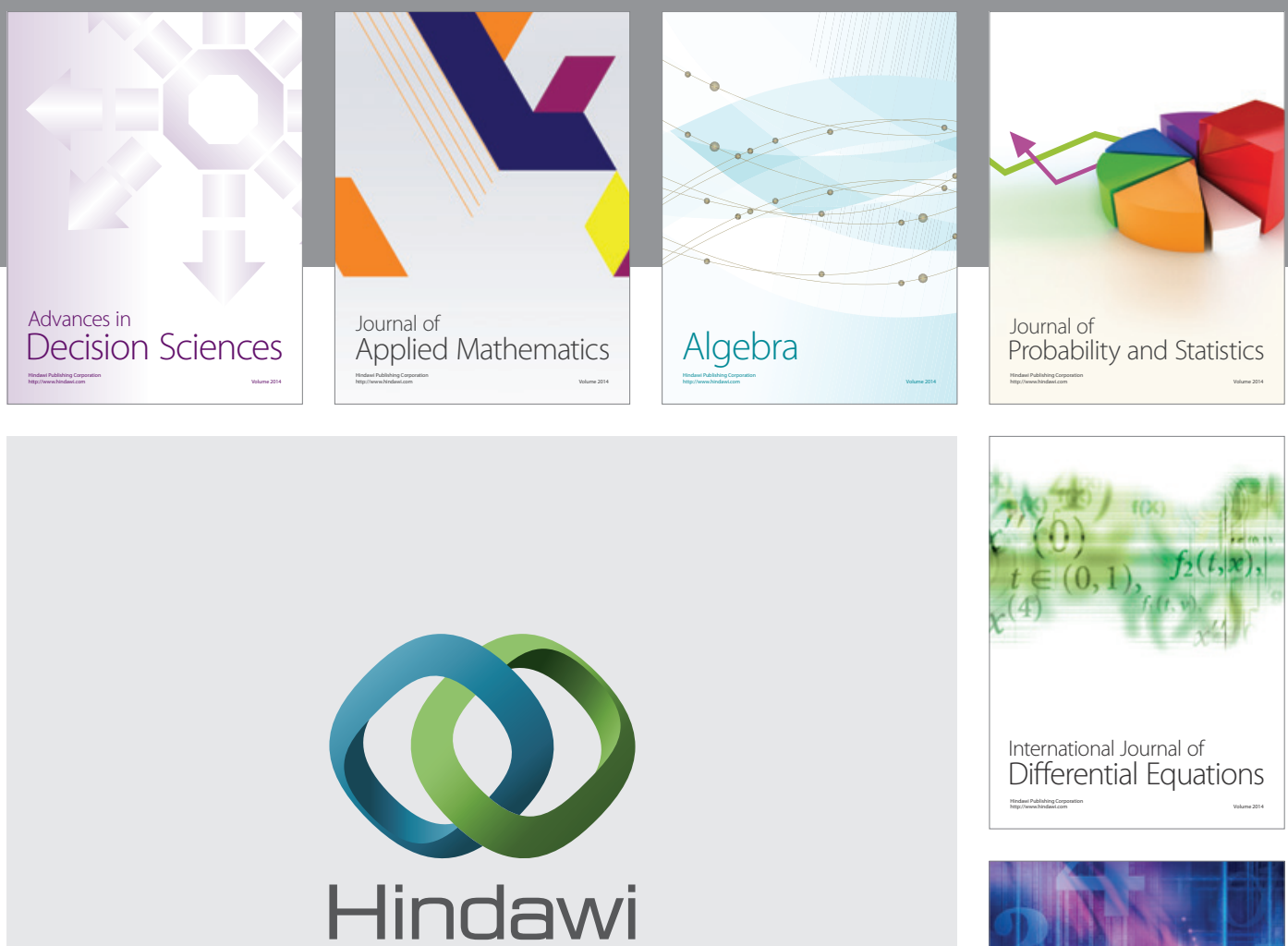

Submit your manuscripts at http://www.hindawi.com
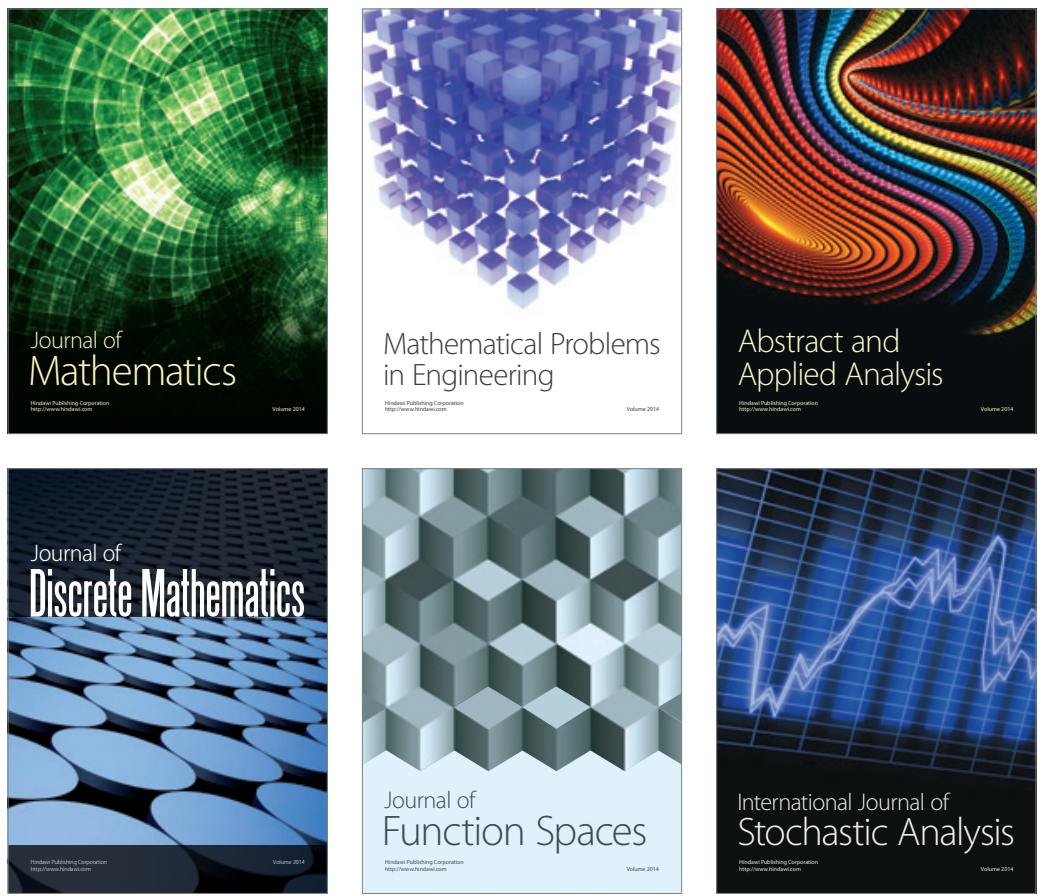

Journal of

Function Spaces

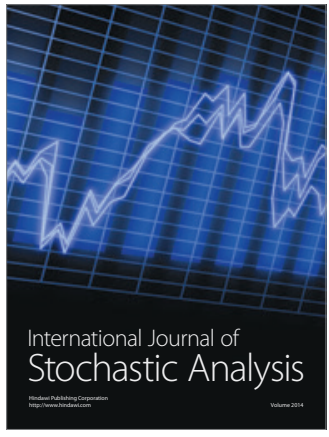

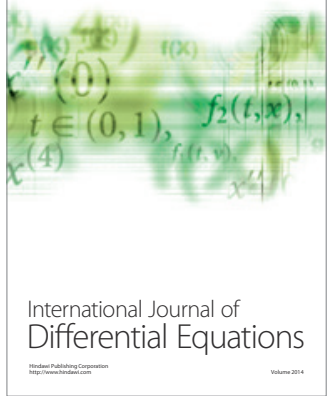
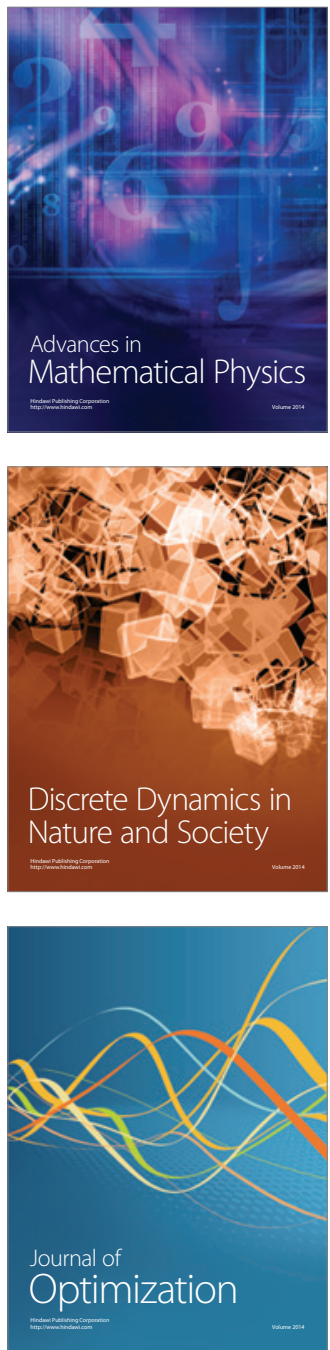